\title{
The need for accessibility to chloride to solubilize insoluble salts may underlie some cases of hypertension
}

Running title: Solubilization of insoluble salts in hypertension

Lipeng Liao ${ }^{1}$, Tao Gan ${ }^{2}$, Yuhan Lin ${ }^{1}$, Chanyuk Lam ${ }^{1}$, Jess Lan Ouyang ${ }^{1}$, Qiuyun Liu $^{1}$

${ }^{1}$ School of Life Sciences, Sun Yat-sen University, Guangzhou 510275, China.

${ }^{2}$ School of Basic Medicine, Gannan Medical University, Ganzhou 34100, Jiangxi, China

$\triangle$ Correspondence: Qiuyun Liu, Ph.D, School of Life Sciences, Sun Yat-sen University, Guangzhou 510275, China. Tel: $\left[\begin{array}{llll}+86 & (020) & 84110296\end{array}\right]$ (lsslqy@mail.sysu.edu.cn). 
Abstract

Hypertension is the greatest risk factor for stroke. It is the most common comorbidity of COVID-19, and may be partly caused by the widespread distribution of insoluble and stiff calcium salts such as calcium oxalate. As an intrinsic defensive mechanism, tissues in hypertensive individuals increase blood pressure to pump $\mathrm{NaCl}$ to inaccessible parts of the body, and chloride, as a strong anion can solubilize insoluble and rigid salts which are stressful to cells.

KEYWORDS: Chloride; Solubilization; Insoluble salts; Hypertension; COVID-19 
Hypertension is the most common comorbidity of COVID-19 [1], and may be triggered in part by the widespread distribution of insoluble and stiff calcium salts such as calcium oxalate in human body. As an endogenous defensive mechanism, tissues in hypertensive individuals increase blood pressure to pump $\mathrm{NaCl}$ to inaccessible parts of the body. Chloride, as a strong anion can solubilize insoluble and rigid salts which are stressful to cells [2]. This is somehow analogous to salting the road in winter to disrupt hydrogen bonding of ice by strong anion $\mathrm{Cl}^{-}$. The proteins of SARS-CoV-2 virus harbor high valine plus glycine content, and the two amino acids potentially possess high affinities to calcium [2-6]. With long carbonyl bond lengths, glycine has been shown to chelate zinc and other divalent cations previously [7]. Valine is also projected to possess secondary chemical bonding [8]. Oxalate is produced from energy metabolism which is robust at low ambient temperature such as winter temperature [2,9]. The slightly lower comorbidity of heart disease and COVID-19 can be attributed to the restricted distribution of calcium oxalate and a large pool of $\mathrm{NaCl}$ in the heart in cardiac disease sufferers [9]. A study suggests a lower COVID-19 risk in hypertensive patients treated with angiotensin-converting enzyme inhibitors or angiotensin receptor blockers compared with calcium channel blockers [1]. To curb SARS-CoV-2 infections, either calcium or oxalate needs to be lowered via the control of protein intake with dietary regimens or energy metabolism via food restriction and winter heating $[2,3,10]$, besides vaccination, passive immunization and other helpful measures. The aforementioned perspective on hypertension could be helpful in the illumination of mechanism underlying some human disorders. Future studies on chloride stress, the calcium oxalate stress, or the antagonism or additivity of the two stresses will further our understanding on the etiology of numerous diseases.

\section{Acknowledgement}

This work was supported by grant from Guangzhou Science and Technology Program (201804010328) to Q. Liu; Natural Science Foundation of Jiangxi Province of China (20181BAB215015) to T. Gan. We thank Yan Shi for editing. 


\section{References}

1. Semenzato L, Botton J, Drouin J, Baricault B, Vabre C, Cuenot F, et al. Antihypertensive Drugs and COVID-19 Risk: A Cohort Study of 2 Million Hypertensive Patients. Hypertension. 2021;77(3):833-42. https://doi.org/10.1161/HYPERTENSIONAHA.120.16314

2. Wan Y, Yan S, Zhang Y, An S, Yang K, Xu H, Gan T, et al. The Pneumonia outbreak: High isoleucine and high valine plus glycine contents are features of the proteins of COVID-19 virus. Preprints. 2020. doi: 10.20944/preprints202002.0289.v5

3. Yan S, Zhang Y, Liu Q. Why COVID-19 virus is so deadly to cancer patients? Eur J Cancer Prev. 2020;29(4):365.

4. An S, Zhang X, Shi Y, Zhang J, Wan Y, Wang Y, et al. High glycine content in TDP-43: a potential culprit in limbic-predominant age-related TDP-43 encephalopathy. J Inter Med Res. 2020;48(6):300060520929853. https://doi.org/10.1177/0300060520929853

5. Qi J, Zhang X, Chen Z, Shi Y, Liu Q. Transgenic proteins rich in valine or glycine are concerns for heart disease patients. Eur J Prev Cardiol. 2018;25(8):883-4. https://doi.org/10.1177/2047487318758111

6. Wan Y, Zhang J, Li X, Wang Y, Liu Q. Cellular states and secondary chemical bonding: a biochemical view of major human diseases. Biochem Insights. 2019;12:1178626419877846. https://doi.org/10.1177/1178626419877846

7. Wang Y, Tang JW, Ma WQ, Feng J, Feng J. Dietary zinc glycine chelate on growth performance, tissue mineral concentrations, and serum enzyme activity in weanling piglets. Biol Trace Elem Res 2010;133(3):325-34. https://doi.org/10.1007/s12011-009-8437-3

8. Zhang X, Ma X, Gan T, Shi Y, Wang Y, Liu Q. Secondary Chemical Bonding between Insoluble Calcium Oxalate and Carbonyl Oxygen Atoms of GLY and VAL Residues Triggers the Formation of A $\beta$ Aggregates and Their Deposition in the Brain. $\begin{array}{lll}\text { ACS Chem 2020;11(24):4007- } & \text { Neurosci. }\end{array}$ 11.https://doi.org/10.1021/acschemneuro.0c00662

9. Zhou Y, Shi Y, Wan Y, Ye ZW, Liu Q. Why the Mediterranean diet lowers the 
risk of heart disease. Eur J Prev Cardiol. 2017;24:1788-9. doi:10.1177/2047487317730653

10. Gan T, Fu M, Wu J, Wen L, Liu Q. How to design carbohydrate diet regimens for heart disease patients. Eur J Prev Cardiol. 2018; 25(9): 979-80. https://doi.org/10.1177/2047487318766602 